\title{
Lipid metabolism of orchiectomised rats was affected by fructose ingestion and the amount of ingested fructose
}

\author{
Satoru Makino, Taro Kishida and Kiyoshi Ebihara* \\ Department of Biological Resources, Faculty of Agriculture, Ehime University, 3-5-7 Tarumi, Matsuyama 790-8566, Japan
}

(Received 6 January 2011 - Revised 5 May 2011 - Accepted 6 June 2011 - First published online 1 August 2011)

\section{Abstract}

We examined whether lipid metabolism in orchiectomised (ORX) rats was affected by fructose ingestion and the amount of ingested fructose. Sucrose was used as a fructose source. Sham-operated and ORX rats were fed one of the following three diets for $28 \mathrm{~d}$ : a maize starch-based diet without sucrose (SU0), a diet by which half or all of maize starch was replaced by sucrose (SU50 or SU100). Body-weight gain and food intake were increased by sucrose ingestion, but decreased by ORX. Plasma total cholesterol concentration was increased by ORX and dose-dependently by sucrose ingestion. Plasma TAG concentration was decreased by ORX, but was increased dose-dependently by sucrose ingestion. Plasma insulin concentration was decreased by ORX, but was not affected by sucrose ingestion. Liver TAG was increased by sucrose ingestion and ORX; however, liver cholesterol concentration was not affected by sucrose ingestion and ORX. The hepatic activity of cholesterol $7 \alpha$-hydroxylase 1 was not affected by sucrose ingestion and ORX; however, faecal excretion of bile acids was decreased. The mRNA level of microsomal TAG transfer protein, which is the gene related to hepatic VLDL production, was increased by ORX and sucrose ingestion. The mRNA level of uncoupling protein-1 was decreased by ORX, but not by sucrose ingestion. Plasma insulin concentration tended to correlate with the level of sterol-regulatory element-binding protein-1c mRNA $(r$ 0.747, $P=0.088$. These results show that lipid metabolism in ORX rats would be affected by the consumption of fructose-rich sweeteners such as sucrose and high-fructose syrup.

Key words: Orchiectomised rats: Sucrose: Plasma lipids: Liver lipids: Bile acids

It is well known that lipid metabolism is influenced by sex hormones in animals and human subjects ${ }^{(1)}$. Males do not have a menopause equivalent, but sex hormones fall with advancing age. Studies in animals and human subjects suggest that androgen deficiency is associated with increased plasma TAG, total cholesterol and LDL-cholesterol.

The type of dietary carbohydrates also influences lipid metabolism. It is well known that lipid metabolism is influenced by high fructose intake ${ }^{(2)}$. Studies based on highfructose $v$. high-glucose diets have shown that high-fructose diets produce an increase in the concentrations of plasma TAG, total cholesterol, VLDL-cholesterol and LDL-cholesterol $^{(3)}$. The availability of fructose increased substantially when it became possible in the 1960s to produce high-fructose syrup economically from maize starch and other starches ${ }^{(4)}$. The most recent available data suggest that fructose consumption is increasing worldwide ${ }^{(5,6)}$. The world average per capita sugar consumption, which does not include high-fructose syrup, has increased from $56 \mathrm{~g} / \mathrm{d}$ in 1986 to $65 \mathrm{~g} / \mathrm{d}$ in $2007^{(7)}$. The average per capita hydroxypropyl starch consumption in Europe has increased from $40 \mathrm{~g} / \mathrm{d}$ in 1985 to $52.4 \mathrm{~g} / \mathrm{d}$ in $2005^{(7)}$. As described earlier, both male sex hormone and fructose influence lipid metabolism. However, less information is available concerning the relationship between male sex hormones and fructose in lipid metabolism. The aim of the present study was to consider the effects of male sex hormones and fructose on endogenous cholesterol metabolism. Therefore, we compared the effects of fructose on lipid metabolism in sham-operated (sham) and orchiectomised (ORX) rats fed a semi-purified diet with or without sucrose. Sucrose was used as a fructose source.

\section{Materials and methods}

\section{Animals and diets}

The present study was approved by the Laboratory Animal Care Committee of Ehime University. Rats were maintained in accordance with the Guidelines for the Care and Use of Laboratory Animals of Ehime University.

\footnotetext{
Abbreviations: CYP7A1, cholesterol 7 $\alpha$-hydroxylase 1; MTP, microsomal TAG transfer protein; ORX, orchiectomised; SREBP, sterol-regulatory elementbinding protein; SU0, maize starch-based diet without sucrose; SU100, diet by which all of maize starch was replaced by sucrose; SU50, diet by which half of maize starch was replaced by sucrose; UCP-1, uncoupling protein-1.
}

*Corresponding author: K. Ebihara, fax +81 899469847 , email ebihara@agr.ehime-u.ac.jp 
Sprague-Dawley male rats (6 months old, $n$ 36) were housed individually in screen-bottomed stainless-steel cages in a room maintained at $23 \pm 1^{\circ} \mathrm{C}$ with a $12 \mathrm{~h}$ light $-12 \mathrm{~h}$ dark cycle (lights on, 07.00-19.00 hours). The rats were acclimatised by feeding them a commercial solid diet (Roden Lab, Diet EQ; PMI International, Brentwood, MO, USA) for $7 \mathrm{~d}$. After acclimatisation, half of the rats were bilaterally ORX under sodium pentobarbital $(30 \mathrm{mg} / \mathrm{kg}$ body weight; Nembutal; Abbott Laboratories, Chicago, IL, USA) and the other half of the rats were sham-operated. Orchiectomy was done through an anterior median incision in the scrotum. Each ductus deferens was isolated, ligated and severed, allowing the testicle to be removed. The rats were fed the commercial solid diet during the $7 \mathrm{~d}$ recovery period, after which ORX and sham-operated rats were randomly divided into three groups ( $n$ 6), respectively, and allowed free access to one of the experimental diets for $28 \mathrm{~d}$. The compositions of each diet are shown in Table $1^{(7)}$. Body weight and food intake were recorded daily for each rat in the morning before the food was replaced.

\section{Sampling and analytical procedures}

Before the rats were killed, faeces were collected from each rat over the final $4 \mathrm{~d}$ of the experimental period. The faeces were freeze-dried, weighed and milled. On the last day of the experimental period, diet was deprived after recording food intake.

A blood sample was collected from the jugular vein using a light diethyl ether anaesthesia on the rats at night. Blood was collected into a blood collection tube (Vacutainer; Becton Dickinson, Franklin Lakes, NJ, USA) that contained heparin as an anticoagulant. Plasma was separated by centrifugation at $1400 \mathrm{~g}$ at $4^{\circ} \mathrm{C}$ for $15 \mathrm{~min}$ and stored at $-50^{\circ} \mathrm{C}$ until analysed. After blood collection, the liver was immediately perfused with cold saline $(\mathrm{NaCl}, 9 \mathrm{~g} / \mathrm{l})$, removed, washed with cold saline, blotted dry on filter paper, weighed and stored at $-50^{\circ} \mathrm{C}$ until analysed. After the liver had been removed, the small intestine was removed. The contents of the small intestine were transferred into a preweighed tube by washing out with physiological saline, freeze-dried and

Table 1. Composition of the experimental diets

\begin{tabular}{lrcc}
\hline & \multicolumn{3}{c}{ Diet $(\mathrm{g} / \mathrm{kg})$} \\
\cline { 2 - 4 } & SU0 & SU50 & SU100 \\
\hline Casein & 200 & 200 & 200 \\
Gelatinised maize starch & 632 & 316 & - \\
Sucrose & 0 & 316 & 632 \\
Cellulose $^{*}$ & 50 & 50 & 50 \\
Soyabean oil & 70 & 70 & 70 \\
AIN-93 mineral mixture† & 35 & 35 & 35 \\
AIN-93 vitamin mixture† & 10 & 10 & 10 \\
L-Cys & 3 & 3 & 3 \\
\hline
\end{tabular}

SU0, maize starch-based diet without sucrose; SU50, diet by which half of maize starch was replaced by sucrose; SU100, diet by which all of maize starch was replaced by sucrose.

* PC200 (Danisco Japan Limited, Tokyo, Japan).

$\dagger$ Based on AIN-93G ${ }^{(7)}$.

¥ The AIN-93 vitamin mixture used in the present study contained $20 \mathrm{~g}$ choline bitartrate/100 g. weighed. The washed small intestine was wiped with a paper towel and returned to the carcass. The stomach, caecum and colon were then removed and opened, and the contents were washed away with physiological saline and returned to the carcass after wiping with a paper towel.

White adipose tissues (perirenal, epididymal and mesenteric adipose tissues) and interscapular brown adipose tissue were removed and weighed. White adipose tissues were returned to the carcass after weighing. The whole carcass was weighed and immediately preserved at $-50^{\circ} \mathrm{C}$ for further analysis.

\section{Biochemical analysis}

The concentrations of total cholesterol, TAG and phospholipids in the plasma were determined enzymatically using commercial diagnostic kits (Cholesterol E-Test Wako, TAG E-Test Wako and Phospholipid C-Test Wako; Wako Pure Chemical Industries, Osaka, Japan). The level of liver total lipids was determined gravimetrically after extraction in accordance with the method of Folch et al. ${ }^{(8)}$. Liver TAG and cholesterol levels were determined enzymatically as described previously ${ }^{(9)}$. Steroids were extracted from the faeces and digestive tract contents (small intestine and caecum) with a chloroform-methanol mixture $(1: 1, \mathrm{v} / \mathrm{v})$ at $70^{\circ} \mathrm{C}$ for $60 \mathrm{~h}$. The concentrations of bile acids in the smallintestinal contents and faeces were determined enzymatically by the 3-dehydrogenase assay method of Sheltaway \& Losowsky $^{(10)}$ using taurocholic acid as a standard. Cholesterol $7 \alpha$-hydroxylase (CYP7A1) activity in the liver was determined according to the method of Ogishima \& Okuda ${ }^{(11)}$.

\section{RNA extraction from the liver and adipose tissue and RT-PCR analysis of gene expression}

Total RNA was extracted from frozen livers and interscapular fat pad for brown adipose tissue in accordance with the method described by Chomczynski \& Sacchi ${ }^{(12)}$. RNA integrity was verified by agarose gel electrophoresis after purification of mRNA using Oligotex-dT30 (Takara Bio, Shiga, Japan). Then, $1 \mu \mathrm{g}$ of mRNA was used for complementary DNA synthesis with 10 units of avian myeloblastosis virus RT (Takara Bio) and $2 \mu \mathrm{l}$ of oligo(dT) primer (Novagen, Inc., Madison, WI, USA) in accordance with the manufacturers' instructions. Expression of mRNA for acyl-coenzyme A:cholesterol acyltransferase 1, acyl-coenzyme A:cholesterol acyltransferase 2, $\beta 3$-adrenergic receptor, ApoB, ApoE, cholesterol 27-hydroxylase, cholesterol $12 \alpha$-hydroxylase, fatty acid synthase, farnesoid $\mathrm{X}$ receptor, 3-hydroxy-3-methylglutaryl coenzyme A reductase, LDL-receptor, liver $\mathrm{X}$ receptor, microsomal TAG transfer protein (MTP), sterol-regulatory element-binding protein-1a (SREBP-1a), sterol-regulatory element-binding protein1c (SREBP-1C), sterol-regulatory element-binding protein-2 (SREBP-2), uncoupling protein-1 (UCP-1) and $\beta$-actin (as a housekeeping gene for normalisation) was determined by real-time monitoring of PCR using a Light Cycler instrument (Roche Diagnostics, Mannheim, Germany). Then, $2 \mu \mathrm{l}$ of complementary DNA were amplified in a total volume of $20 \mu \mathrm{l}$ using the $2 \times$ QuantiTect SYBR Green RTPCR Master 
Mix (Qiagen, Hilden, Germany) and specific primers each at $0.5 \mathrm{M}$. After initial denaturation and activation of the polymerase at $95^{\circ} \mathrm{C}$ for $15 \mathrm{~min}$, cycling was performed for fifty cycles with annealing at the temperatures shown in Table 2 for $25 \mathrm{~s}$, synthesis was performed at $72^{\circ} \mathrm{C}$ for $30 \mathrm{~s}$ and denaturation was performed at $94^{\circ} \mathrm{C}$ for $15 \mathrm{~s}$. Fluorescence was measured at the end of the elongation step at $72^{\circ} \mathrm{C}$. The sequences of the gene-specific primers (Carl Roth, Karlsruhe, Germany) used in the present study are listed in Table 2. Relative gene expression was calculated by using the crossing point of each target gene, with the crossing point of the $\beta$-actin gene used as the reference.

\section{Body composition analysis}

The frozen carcasses were minced using an electric mincing machine (\#22GM-D, Nippon Career Industry Company Limited, Matsuyama, Japan), ground and thoroughly mixed. The moisture content of the carcass was analysed by drying to constant weight at $90^{\circ} \mathrm{C}$ in an oven. Then, minced carcasses were freeze-dried pending the determination of their fat and $\mathrm{N}$ contents. Carcass $\mathrm{N}$ was determined in $250-300 \mathrm{mg}$ samples of dehydrated carcasses using the micro Kjeldahl procedure. Carcass protein content was computed by multiplying the carcass $\mathrm{N}$ content by $6 \cdot 25$. Carcass fat content was in approximately $1.0 \mathrm{~g}$ samples of dehydrated carcasses using a Soxhlet apparatus with chloroform-methanol (2:1).

\section{Statistical analyses}

Data are expressed as means with their standard errors $(n 6)$. To test the significance of the effects of sucrose and ORX, and their interaction, two-way ANOVA (StatView version 4.5;
Abacus Concepts, Berkeley, CA, USA) was used. When significant $F$ ratios were found, individual comparisons were made by the Tukey-Kramer test using the Super ANOVA statistical software package (Abacus Concepts). Differences were considered to be significant at $P<0 \cdot 05$.

\section{Results}

Body-weight gain, food intake, plasma lipids, liver lipids and carcass composition

Body-weight gain, food intake and feed efficiency were increased by sucrose ingestion, but decreased by ORX (Table 3). The weights of perirenal and mesenteric white adipose tissue and interscapular brown adipose tissue were not affected by sucrose ingestion and ORX. The weight of epididymal white adipose tissue was not affected by sucrose ingestion, but decreased by ORX. The body fat percentage was increased by sucrose ingestion, but the body water percentage was decreased by sucrose ingestion, while they were not affected by ORX. The body protein percentage was not affected by sucrose ingestion and ORX.

Plasma total cholesterol and phospholipid concentrations were increased by sucrose ingestion and ORX (Table 4). Plasma TAG concentration was increased by sucrose ingestion, but was decreased by ORX. In sham-operated rats, plasma TAG and phospholipid concentrations were significantly $(P<0.05)$ higher in SU100 diet-fed rats than in SU0 diet-fed rats. Plasma glucose concentration was increased by sucrose ingestion, but was not affected by ORX. Plasma insulin concentration was decreased by ORX, but was not affected by sucrose ingestion. Liver weight was increased by sucrose ingestion, but was not affected by ORX. In sham-operated rats, liver weight was significantly greater in SU100 diet-fed

Table 2. Primer sequences, product sizes and annealing temperatures

\begin{tabular}{|c|c|c|c|c|}
\hline \multirow[b]{2}{*}{ Gene } & \multicolumn{2}{|c|}{ Primer sequence } & \multirow{2}{*}{$\begin{array}{l}\text { Product size } \\
\quad \text { (bp) }\end{array}$} & \multirow{2}{*}{$\begin{array}{l}\text { Annealing temperature } \\
\qquad\left({ }^{\circ} \mathrm{C}\right)\end{array}$} \\
\hline & Sense $\left(5^{\prime} \rightarrow 3^{\prime}\right)$ & Antisense $\left(5^{\prime} \rightarrow 3^{\prime}\right)$ & & \\
\hline ACAT1 & ATG TGG GAA GTA AAT GAA GC & AAA TAC TAG CCA GAC CGA AT & 193 & 53 \\
\hline ACAT2 & CGGTCATGCTGATCCTCTTT & GTGTCACCAGCTCCCAAAAT & 204 & 55 \\
\hline$A p o B$ & TTG ACA CAC TGA AGT TCC TA & ACA TCA AAC CCT GGT ATT AG & 124 & 60 \\
\hline ApoE & AAC CGC TTC TGG GAT TAC CT & CCA GCT CCT TTT TGT ATG CC & 145 & 55 \\
\hline CYP7A1 & CTG CAA ACT GAT GGG GAA AT & TGG GTC AAT GCT TCT GTG TC & 145 & 60 \\
\hline CYP8B1 & ATG AAG GCT GTG CGA GAG & TCT CTT CCA TCA CGC TGT C & 127 & 59 \\
\hline CYP27 & CTC AAG AGA CTG TCG GCA C & CTT CCT GGA TCT CTG GGT T & 114 & 57 \\
\hline FAS & AGTACCACGGCAATGTGA & ACGATGGTCACCCTCAAT & 134 & 53 \\
\hline FXR & CGT TCA GCG GAG ATT TTC AA & ATT TTC AGC TCC CCG ACA CT & 140 & 57 \\
\hline HMG-CoA reductase & GCT GGT GAG TTG TCC TTG AT & CTT CTT GGT GCA TGT TCC CT & 117 & 60 \\
\hline LDL-receptor & CTT GCC CTG ATG GTA TGC TA & CTT GCG CTT CAG TGA CAC A & 137 & 60 \\
\hline$L X R \alpha$ & TGC TAA TGA AGC TGG TGA & AGA GGA CAC GGA GAA ACA GT & 144 & 57 \\
\hline MTP & AGCGACATCACAGTGGACTC & GGCTCTCTTTTTCTTTCTCCGA & 223 & 62 \\
\hline$R X R \alpha$ & CAA ACA TGG GGC TGA ACC & AGC AGG ATG ACC TGG TCG T & 148 & 57 \\
\hline SREBP-1a & CCG AGA TGT GCG AAC TGG AC & TGT CTC CTG TCT CAC CCC CA & 129 & 55 \\
\hline SREBP-1C & GGAGCCATGGATTGCACATT & AGGAAGGCTTCCAGAGAGGA & 191 & 53 \\
\hline SREBP-2 & CACAATATCATTGAAAAGCGCTACG & TTTTTCTGATTGGCCAGCTTCAGCA & 200 & 55 \\
\hline$U C P-1$ & TATAGCCACCACAGAAAGC & GGACGTCATCTGCCAGTAT & 149 & 55 \\
\hline$\beta 3$-Adrenoreceptor & TCTTCTGTGCAGCTACGCT & GTCCTTCATGTGGGAAATGG & 144 & 55 \\
\hline$\beta$-Actin & CTATGAGCTGCCTGACGGTC & AGTTTCATGGATGCACAGG & 115 & 53 \\
\hline
\end{tabular}

ACAT, cholesterol acyltransferase; CYP7A1, cholesterol $7 \alpha$-hydroxylase 1; CYP8B1, cholesterol 12 $\alpha$-hydroxylase; CYP27, cholesterol 27-hydroxylase; FAS, fatty acid synthase; FXR, farnesoid X receptor; HMG-CoA, 3-hydroxy-3-methylglutaryl coenzyme A; $L X R \alpha$, liver X receptor- $\alpha$; MTP, microsomal TAG transfer protein; RXR $\alpha$, retinoid $\mathrm{X}$ receptor- $\alpha$; SREBP, sterol-regulatory element-binding protein; UCP-1, uncoupling protein-1. 
WS British Journal of Nutrition

Table 3. Effects of sucrose ingestion and orchiectomy (ORX) on body-weight gain, food intake, adipose tissue weight and body composition $\dagger$

(Mean values with their standard errors, $n 6$ )

\begin{tabular}{|c|c|c|c|c|c|c|c|c|c|c|c|c|c|c|c|}
\hline & \multicolumn{6}{|c|}{ Sham } & \multicolumn{6}{|c|}{ ORX } & & & \\
\hline & \multicolumn{2}{|l|}{ C } & \multicolumn{2}{|c|}{ su50 } & \multicolumn{2}{|c|}{ SU100 } & \multicolumn{2}{|l|}{ C } & \multicolumn{2}{|l|}{ su50 } & \multicolumn{2}{|c|}{ SU100 } & \multicolumn{3}{|c|}{ Two-way ANOVA } \\
\hline & Mean & SE & Mean & SE & Mean & SE & Mean & $\mathrm{SE}$ & Mean & SE & Mean & SE & Diet & ORX & Interaction \\
\hline Initial body weight & 553 & 13 & 545 & 14 & 551 & 18 & 543 & 15 & 542 & 15 & 544 & 17 & 0.956 & 0.504 & 0.954 \\
\hline Final body weight & 573 & 19 & 596 & 17 & 591 & 22 & 549 & 15 & 565 & 15 & 562 & 17 & 0.526 & 0.063 & 0.974 \\
\hline Body-weight gain (g/4 weeks) & $19^{a, b}$ & 8 & $51^{\mathrm{C}}$ & 5 & $40^{\mathrm{b}, \mathrm{c}}$ & 8 & $7^{\mathrm{a}}$ & 4 & $23^{a, b}$ & 1 & $18^{\mathrm{a}, \mathrm{b}}$ & 8 & 0.002 & $<0.001$ & 0.464 \\
\hline Food intake (g/4 weeks) & $496^{\mathrm{a}}$ & 28 & $591^{\mathrm{b}}$ & 21 & $561^{a, b}$ & 23 & $482^{\mathrm{a}}$ & 9 & $504^{\mathrm{a}}$ & 12 & $524^{a, b}$ & 17 & 0.009 & 0.007 & 0.172 \\
\hline Feed efficiency & $0.036^{a, b}$ & 0.013 & $0.085^{\mathrm{c}}$ & 0.007 & $0.069^{b, c}$ & 0.011 & $0.014^{\mathrm{a}}$ & 0.009 & $0.045^{a, b, c}$ & 0.003 & $0.033^{a, b}$ & 0.014 & 0.002 & $<0.001$ & 0.656 \\
\hline \multicolumn{16}{|l|}{ White adipose tissue (g) } \\
\hline Perirenal & 15.9 & 0.9 & 17.9 & 1.4 & 17.7 & $2 \cdot 0$ & 14.9 & 0.8 & $16 \cdot 2$ & 1.3 & $16 \cdot 0$ & $2 \cdot 0$ & 0.598 & 0.369 & 0.942 \\
\hline Epididymal & $12 \cdot 6$ & 0.8 & $14 \cdot 0$ & $1 \cdot 1$ & $15 \cdot 4$ & $2 \cdot 0$ & $12 \cdot 0$ & $1 \cdot 1$ & $12 \cdot 4$ & $1 \cdot 2$ & 10.4 & $1 \cdot 2$ & 0.754 & 0.031 & 0.229 \\
\hline Mesenteric & 11.3 & 1.2 & $12 \cdot 3$ & 0.8 & $12 \cdot 1$ & 1.1 & $10 \cdot 6$ & 0.6 & 11.3 & 1.0 & $10 \cdot 1$ & 1.2 & 0.665 & 0.137 & 0.784 \\
\hline Brown adipose tissue (g) & 0.497 & 0.018 & 0.447 & 0.034 & 0.455 & 0.065 & 0.424 & 0.046 & 0.397 & 0.060 & 0.591 & 0.020 & 0.359 & 0.579 & 0.261 \\
\hline \multicolumn{16}{|l|}{ Body composition (\%) } \\
\hline Moisture & 58.4 & 0.8 & 58.0 & 0.8 & 55.4 & 0.5 & $59 \cdot 0$ & 1.0 & 57.7 & 0.9 & 56.5 & 0.9 & 0.010 & 0.788 & 0.840 \\
\hline Fat & $17 \cdot 4^{\mathrm{a}, \mathrm{b}}$ & 1.2 & $17 \cdot 0^{\mathrm{a}, \mathrm{b}}$ & 1.2 & $21 \cdot 6^{\mathrm{b}}$ & 1.7 & $15 \cdot 6^{\mathrm{a}}$ & 1.0 & $17 \cdot 6^{a, b}$ & 1.0 & $18 \cdot 8^{\mathrm{a}, \mathrm{b}}$ & 1.0 & 0.011 & 0.181 & 0.351 \\
\hline Protein & 20.9 & $0 . \overline{5}$ & 21.0 & 0.3 & 20.5 & 0.5 & $21 \cdot 4$ & 0.5 & $21 \cdot 6$ & 0.6 & $20 \cdot 8$ & 0.7 & 0.441 & 0.306 & 0.933 \\
\hline
\end{tabular}

C, control; SU0, maize starch-based diet without sucrose; SU50, diet by which half of maize starch was replaced by sucrose; SU100, diet by which all of maize starch was replaced by sucrose.

a,b,c Mean values within a row with unlike superscript letters were significantly different $(P<0.05)$

$\neq$ Significant effect $(P<0.05)$ of dietary sucrose ingestion (sucrose) and ORX, and significant effect of the interaction between dietary sucrose ingestion and ORX (sucrose $\times$ ORX).

Table 4. Effects of sucrose ingestion and orchiectomy (ORX) on body-weight gain, food intake, adipose tissue weight and body compositiont

(Mean values with their standard errors, $n 6$ )

\begin{tabular}{|c|c|c|c|c|c|c|c|c|c|c|c|c|c|c|c|}
\hline & \multicolumn{6}{|c|}{ Sham } & \multicolumn{6}{|c|}{ ORX } & & & \\
\hline & \multicolumn{2}{|c|}{$\mathrm{C}$} & \multicolumn{2}{|c|}{ SU50 } & \multicolumn{2}{|c|}{ SU100 } & \multicolumn{2}{|c|}{ C } & \multicolumn{2}{|c|}{ SU50 } & \multicolumn{2}{|c|}{ SU100 } & \multicolumn{3}{|c|}{ Two-way ANOVA $\ddagger$} \\
\hline & Mean & $\mathrm{SE}$ & Mean & SE & Mean & SE & Mean & SE & Mean & $\mathrm{SE}$ & Mean & SE & Diet & ORX & Interaction \\
\hline \multicolumn{16}{|l|}{ Plasma lipid (mmol/l) } \\
\hline Total cholesterol & 2.32 & 0.21 & 2.78 & 0.18 & $2 \cdot 70$ & 0.14 & $2 \cdot 82$ & 0.11 & 2.92 & 0.20 & 2.97 & 0.13 & 0.042 & 0.019 & 0.554 \\
\hline TAG & $1.02^{\mathrm{a}}$ & 0.05 & $1.45^{\mathrm{a}, \mathrm{b}}$ & 0.11 & $1.60^{\mathrm{b}}$ & 0.21 & $0.99^{\mathrm{a}}$ & 0.06 & $1 \cdot 10^{\mathrm{a}, \mathrm{b}}$ & 0.11 & $1 \cdot 11^{\mathrm{a}, \mathrm{b}}$ & 0.12 & 0.013 & 0.005 & 0.130 \\
\hline Phospholipid & $1.65^{\mathrm{a}}$ & $0 \cdot 10$ & $1.91^{\mathrm{a}, \mathrm{b}}$ & 0.07 & $2 \cdot 17^{\mathrm{b}}$ & 0.11 & $1.94^{\mathrm{a}, \mathrm{b}}$ & 0.07 & $2 \cdot 26^{a, b}$ & 0.08 & $2 \cdot 28^{\mathrm{b}}$ & 0.11 & 0.002 & 0.011 & 0.531 \\
\hline Plasma glucose $(\mathrm{mmol} / \mathrm{l})$ & $2.99^{a}$ & 0.21 & $3 \cdot 61^{a, b}$ & 0.15 & $4.07^{b}$ & 0.18 & $3 \cdot 28^{\mathrm{a}}$ & 0.22 & $4 \cdot 14^{\mathrm{b}}$ & 0.09 & $3.98^{\mathrm{b}}$ & 0.19 & $<0.001$ & 0.058 & 0.142 \\
\hline \multicolumn{16}{|l|}{ Liver weight } \\
\hline $\mathrm{g}$ & 11.6 & 0.5 & 13.7 & 0.6 & 14.4 & 0.10 & $11 \cdot 2$ & 0.5 & $12 \cdot 9$ & 0.7 & $13 \cdot 4$ & 1.0 & 0.007 & 0.223 & 0.901 \\
\hline$g / 100 \mathrm{~g} \mathrm{BW}$ & $2.04^{a}$ & 0.03 & $2 \cdot 30^{a, b}$ & 0.07 & $2 \cdot 42^{\mathrm{b} *}$ & 0.13 & $2 \cdot 04^{a}$ & 0.05 & $2 \cdot 29^{a, b}$ & 0.13 & $2 \cdot 30^{\mathrm{a}, \mathrm{b}}$ & 0.11 & 0.007 & 0.223 & 0.901 \\
\hline \multicolumn{16}{|l|}{ Liver lipids } \\
\hline Total lipids (mg/g per liver) & 74 & 8.5 & 96 & $13 \cdot 2$ & 85 & $13 \cdot 3$ & 83 & 7 & 113 & $18 \cdot 3$ & 124 & $28 \cdot 6$ & 0.200 & 0.123 & 0.650 \\
\hline Cholesterol ( $\mu \mathrm{mol} / \mathrm{g}$ liver) & 5.39 & 0.74 & 6.53 & 0.58 & 6.26 & 0.70 & $6 \cdot 12$ & 0.56 & 8.94 & 1.61 & 7.00 & 0.69 & 0.101 & 0.085 & 0.560 \\
\hline TAG $(\mu \mathrm{mol} / \mathrm{g}$ liver) & 39.0 & 7.7 & $52 \cdot 8$ & 11.7 & $40 \cdot 3$ & 9.0 & 38.6 & $5 \cdot 9$ & 68.0 & 13.8 & 49.4 & $10 \cdot 7$ & 0.106 & 0.343 & 0.740 \\
\hline
\end{tabular}

C, control; SU0, maize starch-based diet without sucrose; SU50, diet by which half of maize starch was replaced by sucrose; SU100, diet by which all of maize starch was replaced by sucrose; BW, body weight.

${ }_{a, b}$ Mean values within a row with unlike superscript letters were significantly different $(P<0.05)$

t Sham-operated and ORX rats were fed the experimental diets for $28 \mathrm{~d}$.

I Significant effect $(P<0.05)$ of dietary sucrose ingestion and ORX, and significant effect of the interaction between dietary sucrose ingestion and ORX (sucrose $\times$ ORX). 
rats than in SU0 diet-fed rats. The concentrations of total lipids, cholesterol and TAG in the liver were not affected by sucrose ingestion and ORX; however, the concentration of cholesterol in the liver tended to increase by ORX $(P=0.085)$.

Hepatic cholesterol $7 \alpha$-hydroxylase 1 activity, bile acids in intestinal contents and faecal excretion of bile acids

The activity of CYP7A1 in the liver and dry weight of smallintestinal contents were not affected by diet and ORX (Table 5). The amount of bile acids in the small-intestinal contents was decreased by sucrose ingestion, but was not affected by ORX. Faecal dry weight was increased by sucrose ingestion, but decreased by ORX. Faecal excretion of bile acids was decreased by sucrose ingestion and ORX.

\section{Hepatic gene expression}

The levels of cholesterol 12 $\alpha$-hydroxylase, LDL-receptor, SREBP-2, liver $\mathrm{X}$ receptor and fatty acid synthase mRNA in the liver were not affected by sucrose ingestion and ORX (Table 6). The levels of ApoB, cholesterol acyltransferase 2, farnesoid X receptor, 3-hydroxy-3-methylglutaryl coenzyme A reductase, retinoid $X$ receptor- $\alpha$, SREBP-1a and SREBP-1c mRNA in the liver were increased by sucrose ingestion, and they were significantly higher in SU100 diet-fed rats than in SU0 diet-fed rats, but they were not affected by ORX. The level of cholesterol acyltransferase $1 \mathrm{mRNA}$ in the liver was increased by sucrose ingestion and ORX, and was significantly higher in SU100 diet-fed rats than in SU0 diet-fed rats. The level of MTP mRNA in the liver was increased by sucrose ingestion and ORX, and was significantly higher in SU100 diet-fed rats than in SU0 and SU50 diet-fed rats. The levels of cholesterol 27-hydroxylase mRNA in the liver tended to increase by ORX, but were not affected by sucrose ingestion.

\section{Brown adipocyte gene expression}

The level of UCP-1 mRNA tended to decrease by ORX, but was not affected by sucrose ingestion (Table 7). The levels of $\beta 3$-adrenergic receptor were not affected by sucrose ingestion and ORX.

\section{Discussion}

In the present study, ORX caused a significant reduction in food intake, which is in agreement with the results of other researchers ${ }^{(13,14)}$. ORX decreases food intake by decreasing meal frequency ${ }^{(15)}$. The mechanisms underlying this effect are not well understood ${ }^{(16)}$. However, the effects of gonadectomy on eating in rats and mice are apparently due to testosterone in males ${ }^{(17)}$. Anabolic steroids, which have testosterone-like effects, have been shown to increase appetite and food intake in human subjects ${ }^{(18)}$. The decline in testosterone levels in older males leads to increased leptin levels and this may explain the greater decline in food intake with ageing in males ${ }^{(19)}$. Leptin inhibits food intake by acting in the appetite control centres of the brain. 
Table 6. Effects of sucrose ingestion and orchiectomy (ORX) on the hepatic mRNA levels of genes upon lipid metabolism* (Mean values with their standard errors, $n 6$ )

\begin{tabular}{|c|c|c|c|c|c|c|c|c|c|c|c|c|c|c|c|}
\hline \multirow[b]{3}{*}{ Gene } & \multicolumn{6}{|c|}{ Sham } & \multicolumn{6}{|c|}{ ORX } & & & \\
\hline & \multicolumn{2}{|c|}{ C } & \multicolumn{2}{|c|}{ SU50 } & \multicolumn{2}{|c|}{ SU100 } & \multicolumn{2}{|c|}{ C } & \multicolumn{2}{|c|}{ SU50 } & \multicolumn{2}{|c|}{ SU100 } & \multicolumn{3}{|c|}{ Two-way ANOVA† } \\
\hline & Mean & SE & Mean & SE & Mean & SE & Mean & SE & Mean & SE & Mean & SE & Diet & ORX & Interaction \\
\hline$A p o B$ & $1.09^{\mathrm{a}}$ & 0.12 & $1.55^{a, b}$ & 0.07 & $2 \cdot 45^{a, b}$ & 0.62 & $1.07^{\mathrm{a}}$ & 0.04 & $1.83^{\mathrm{a}, \mathrm{b}}$ & 0.29 & $2.94^{\mathrm{b}}$ & 0.65 & 0.001 & 0.457 & 0.802 \\
\hline ApoE & 1.04 & 0.05 & 1.06 & 0.03 & 1.22 & 0.09 & 1.09 & 0.07 & 0.99 & 0.06 & $1.62^{\mathrm{b}}$ & 0.08 & $<0.001$ & 0.032 & 0.004 \\
\hline ACAT1 & $1.05^{\mathrm{a}}$ & 0.04 & $1.01^{\mathrm{a}}$ & 0.04 & $1.37^{\mathrm{b}}$ & 0.09 & $0.95^{\mathrm{a}}$ & 0.03 & $0.98^{\mathrm{a}}$ & 0.06 & $1 \cdot 17^{\mathrm{a}, \mathrm{b}}$ & 0.03 & $<0.001$ & 0.023 & 0.288 \\
\hline ACAT2 & $0.96^{\mathrm{a}}$ & 0.06 & $1 \cdot 21^{\mathrm{a}, \mathrm{b}}$ & 0.07 & $1 \cdot 39^{b}$ & 0.08 & $1 \cdot 13^{a, b}$ & 0.04 & $1 \cdot 20^{\mathrm{a}, \mathrm{b}}$ & 0.11 & $1 \cdot 34^{\mathrm{b}}$ & 0.04 & $<0.001$ & 0.596 & 0.294 \\
\hline CYP7A1 & $1 \cdot 16$ & 0.23 & 0.67 & 0.17 & 1.43 & 0.28 & 0.82 & 0.13 & 0.42 & 0.09 & 1.28 & 0.40 & 0.015 & 0.264 & 0.936 \\
\hline CYP8B1 & 0.96 & 0.14 & 0.80 & 0.04 & 0.92 & 0.07 & 0.90 & 0.07 & 0.85 & $0 \cdot 10$ & 0.83 & 0.06 & 0.534 & 0.588 & 0.699 \\
\hline CYP27 & $0.87^{\mathrm{a}}$ & 0.06 & $0.90^{\mathrm{a}}$ & 0.09 & $0.88^{\mathrm{a}}$ & 0.06 & $1 \cdot 00^{a, b}$ & 0.05 & $1.00^{\mathrm{a}, \mathrm{b}}$ & 0.08 & $1 \cdot 26^{\mathrm{b}}$ & 0.03 & 0.124 & 0.001 & 0.099 \\
\hline FAS & 0.77 & 0.11 & 1.18 & 0.27 & 1.19 & 0.33 & 1.17 & 0.15 & 0.86 & 0.09 & 1.57 & 0.31 & 0.205 & 0.394 & 0.236 \\
\hline FXR & $1.07^{\mathrm{a}}$ & 0.04 & $1 \cdot 22^{\mathrm{a}, \mathrm{b}}$ & 0.09 & $1.60^{\mathrm{b}}$ & 0.13 & $1.09^{\mathrm{a}}$ & 0.07 & $1 \cdot 25^{\mathrm{a}, \mathrm{b}}$ & 0.13 & $1 \cdot 50^{\mathrm{a}, \mathrm{b}}$ & 0.12 & $<0.001$ & 0.821 & 0.777 \\
\hline HMG-CoA reductase & $1 \cdot 01^{\mathrm{a}, \mathrm{b}}$ & 0.10 & $0.85^{a, b}$ & 0.07 & $1 \cdot 27^{\mathrm{a}, \mathrm{b}}$ & 0.13 & $0.74^{a}$ & 0.06 & $1 \cdot 11^{a, b}$ & 0.13 & $1.40^{\mathrm{b}}$ & 0.24 & 0.006 & 0.766 & 0.154 \\
\hline LDL-receptor & 0.77 & 0.11 & $1 \cdot 18$ & 0.27 & 1.19 & 0.33 & $1 \cdot 17$ & 0.15 & 0.86 & 0.09 & 1.57 & 0.31 & 0.205 & 0.394 & 0.236 \\
\hline$L X R \alpha$ & 1.03 & 0.04 & 1.01 & 0.02 & $1 \cdot 11$ & 0.06 & 0.98 & 0.03 & 0.99 & 0.09 & 1.06 & 0.04 & 0.219 & 0.379 & 0.967 \\
\hline MTP & $1.04^{\mathrm{a}}$ & 0.06 & $1.02^{\mathrm{a}}$ & 0.05 & $1.55^{\mathrm{b}, \mathrm{c}}$ & 0.08 & $1.05^{\mathrm{a}}$ & 0.04 & $1 \cdot 28^{\mathrm{a}, \mathrm{b}}$ & 0.07 & $1.72^{\mathrm{c}}$ & 0.11 & $<0.001$ & 0.027 & 0.238 \\
\hline$R X R \alpha$ & $0.99^{a, b}$ & 0.15 & $1 \cdot 15^{\mathrm{a}, \mathrm{b}, \mathrm{c}}$ & 0.12 & $1 \cdot 60^{\mathrm{b}, \mathrm{c}}$ & 0.18 & $1 \cdot 28^{\mathrm{a}, \mathrm{b}, \mathrm{c}}$ & 0.10 & $0.96^{\mathrm{a}}$ & 0.13 & $1.69^{\mathrm{c}}$ & 0.17 & $<0.001$ & 0.586 & 0.269 \\
\hline SREBP-1a & $0.99^{\mathrm{a}}$ & 0.27 & $1 \cdot 17^{\mathrm{a}, \mathrm{b}}$ & 0.20 & $2 \cdot 03^{\mathrm{C}}$ & 0.20 & $0.90^{\mathrm{a}}$ & 0.10 & $0.84^{\mathrm{a}}$ & 0.13 & $1.86^{\mathrm{b}, \mathrm{c}}$ & 0.16 & $<0.001$ & 0.188 & 0.789 \\
\hline SREBP-1C & $0.99^{\mathrm{a}}$ & 0.27 & $1 \cdot 17^{\mathrm{a}, \mathrm{b}}$ & 0.20 & $1.88^{\mathrm{b}}$ & 0.27 & $0.90^{\mathrm{a}}$ & $0 \cdot 10$ & $0.84^{\mathrm{a}}$ & 0.13 & $1 \cdot 40^{\mathrm{a}, \mathrm{b}}$ & 0.16 & 0.002 & 0.075 & 0.622 \\
\hline SREBP-2 & 0.80 & 0.19 & 0.94 & 0.11 & 0.90 & 0.14 & 0.91 & 0.13 & 0.87 & 0.11 & 0.96 & 0.21 & 0.869 & 0.849 & 0.833 \\
\hline
\end{tabular}

C, control; SU0, maize starch-based diet without sucrose; SU50, diet by which half of maize starch was replaced by sucrose; SU100, diet by which all of maize starch was replaced by sucrose; $A C A T$, cholesterol acyltransferase; CYP7A1, cholesterol 7 $\alpha$-hydroxylase 1; CYP8B1, cholesterol 12 $\alpha$-hydroxylase; CYP27, cholesterol 27-hydroxylase; FAS, fatty acid synthase; FXR, farnesoid X receptor; HMG-CoA, 3-hydroxy-3-methylglutaryl coenzyme A; $L X R \alpha$, liver X receptor- $\alpha$; MTP, microsomal TAG transfer protein; $R X R \alpha$, retinoid X receptor- $\alpha$; SREBP, sterol-regulatory element-binding protein.

a,b,c Mean values within a row with unlike superscript letters were significantly different $(P<0.05)$.

* Sham-operated and ORX rats were fed the experimental diets for $28 \mathrm{~d}$.

† Significant effect $(P<0.05)$ of dietary sucrose ingestion and ORX, and significant effect of the interaction between dietary sucrose and ORX (sucrose $\times$ ORX). 
Therefore, the proposed mechanism for appetite decreased by ORX may be through an increase in circulating leptin. Leptin is manufactured primarily in the adipocytes of white adipose tissue, and the level of circulating leptin is directly proportional to the total amount of fat in the body. Testosterone is a fat-reducing hormone ${ }^{(20)}$. Plasma leptin concentrations have a strong positive correlation with body $\mathrm{fat}^{(21)}$. However, in the present study, the weight of bilateral epididymal fat pads to be an important white adipose tissue was decreased by ORX, and the body fat mass tended to be decreased by ORX $(P=0 \cdot 078)$. Moreover, the concentration of plasma insulin was significantly decreased by ORX. It has been shown that fasting serum leptin and insulin concentrations are highly correlated ${ }^{(22)}$. We did not measure plasma leptin concentration in the present study; however, in our previous study on rats fed the SU0 diet, plasma leptin concentration was significantly increased by ORX (8.83 (SE 0.23$) \mathrm{ng} / \mathrm{ml}$ for ORX rats and 7.21 (SE 0.42$) \mathrm{ng} / \mathrm{ml}$ for sham rats; K Ebihara and S Makino, unpublished results), which agrees with the result of Pinilla et al. ${ }^{(23)}$. On the other hand, plasma leptin half-life was shortened by $\mathrm{ORX}^{(24)}$. Leptin is also secreted by several other tissues such as the stomach, placenta and brain as well as white adipose tissue ${ }^{(25)}$. Though the reason why plasma leptin concentration was increased by ORX with decreasing abdominal fat tissues, and shortened half-life of leptin is at present unclear, an increased leptin concentration might depend on leptin secreted from several other tissues except white adipose tissue. It is certain that leptin plays an important role in the regulation of food intake; however, factors other than leptin also might take part in the food intake decreased by ORX.

ORX also caused a significant reduction in body-weight gain. Body-weight gain tended to be a positive correlation with the weight of body fat $(r 0.402, P=0.073)$ and the body fat mass:body protein mass ratio $(r 0 \cdot 462, P=0 \cdot 053)$. Testosterone stimulates lipolysis by increasing the number of lipolytic $\beta 3$-adrenergic receptor ${ }^{(20)}$. However, in the present study, the level of $\beta 3$-adrenergic receptor mRNA of brown adipose tissue was not affected by ORX. UCP-1 is responsible for dissipating energy as heat instead of generating ATP from the oxidation of NEFA, and implicates for the regulation of total body fat. The level of UCP-1 mRNA of brown adipose tissue was decreased by ORX. Therefore, the decrease in body weight depends on the decrease in body fat mass, and it might depend on the increase in energy expenditure and the decrease in food intake.

Body-weight gain, food intake and body fat mass were increased by the ingestion of sucrose not only in sham-rats but also in ORX rats. There are many studies in rats reporting that sucrose-induced overeating led to obesity ${ }^{(26,27)}$, which might occur because sucrose stimulates the appetite and leads to excess consumption of dietary energy. High consumption of sucrose led to the accumulation of adipose tissue $^{(28)}$. Body-weight gain was a strong positive correlation with food intake ( $r 0.919, P=0.0025)$, which shows that the increases in body-weight gain and body mass fat depend on an increase in food intake. Sucrose is a disaccharide made of glucose and fructose. The consumption of diets high in 
fructose increased de novo lipogenesis ${ }^{(29)}$. Lane \& Cha ${ }^{(30)}$ explored the suggested link between the consumption of fructose and increased food intake in rats. Fructose was shown to result in decreased circulating leptin ${ }^{(31)}$. Therefore, the increases in food intake and body fat mass by sucrose ingestion might depend on fructose in sucrose.

Plasma TAG concentration was increased by sucrose ingestion, but was decreased by ORX. MTP plays a critical role in the secretion of VLDL from the liver and is an important factor for the assembly of TAG-rich lipoproteins ${ }^{(32,33)}$. The level of SREBP-1c mRNA tended to decrease by ORX $(P=0.075)$; however, fatty acid synthase mRNA level and liver TAG concentration was not affected by ORX. Therefore, a decreased synthesis of fatty acid in the liver might explain the decreased plasma TAG concentration in ORX rats. However, some studies in animals and human subjects have suggested that androgen deficiency is associated with increased plasma $\mathrm{TAG}^{(34,35)}$.

Plasma total cholesterol concentration was increased by ORX, which agrees with the results of Haug \& Høstmark ${ }^{(32)}$ and Traish et al. ${ }^{(36)}$. The levels of 3-hydroxy-3-methylglutaryl coenzyme A reductase and SREBP-2 were not affected by ORX, but liver cholesterol concentration tended to be increased by ORX $(P=0 \cdot 085)$. ApoE plays a major role in systemic cholesterol metabolism by serving as a ligand for removal of cholesterol-laden plasma lipoproteins by hepatic receptors. The level of apoE mRNA was increased by ORX. The activity of hepatic CYP7A1 was not affected by ORX; however, the amount of bile acids in the intestinal contents and the faecal excretion of bile acids tended to decrease $(P=0 \cdot 131)$ and was significantly decreased by ORX. Therefore, the increase in plasma cholesterol by ORX might depend on the decreased conversion from cholesterol to bile acids in the liver.

It is known that growth hormone $(\mathrm{GH})$ plays an important role in the regulation of lipid metabolism ${ }^{(37,38)}$. ORX decreased GH levels ${ }^{(39)}$. Fructose stimulates GH secretion ${ }^{(40)}$. SREBP-1c is a possible mediator of the action of $\mathrm{GH}^{(41)}$. Fructose is a stronger inducer of SREBP- $1 \mathrm{c}^{(42)}$. In the present study, the concentration of MTP mRNA was increased by ORX and sucrose ingestion. The level of SREBP-1c mRNA was increased by sucrose ingestion and tended to decrease by ORX. On the other hand, GH stimulates both CYP7A1 activity and faecal bile acid excretion not only in GH-deficient animals, but also in normal young rats ${ }^{(43)}$. However, in the present study, CYP7A1 activity was not affected by ORX and sucrose ingestion, and faecal bile acid excretion was decreased by ORX and sucrose ingestion. Therefore, ORX and sucrose ingestion might influence each other through $\mathrm{GH}$, and regulate lipid metabolism. However further studies are necessary to discuss a relationship or interaction between the role of male hormone and fructose ingestion in lipid metabolism.

In both sham-operated and ORX rats, plasma TAG and cholesterol concentrations increased by sucrose ingestion, and liver TAG and cholesterol concentrations tended to be increased by sucrose ingestion $(P=0 \cdot 106$ and $P=0 \cdot 101)$. Sucrose is a dimer of fructose and glucose. Numerous studies have reported that dietary fructose induces hyperlipidaemia $^{(44-46)}$. Therefore, the increases in plasma TAG and cholesterol concentrations by sucrose ingestion would depend on fructose in sucrose.

In conclusion, lipid metabolism of rats was affected by ORX, sucrose ingestion and the amount of ingested sucrose. Plasma total cholesterol concentration was increased by ORX and dose-dependently by sucrose ingestion. Plasma TAG concentration was decreased by ORX, but was increased dose-dependently by sucrose ingestion. Liver TAG was increased by sucrose ingestion and ORX; however, liver cholesterol concentration was not affected by sucrose ingestion and ORX. The changes in lipid metabolism were influenced by sucrose ingestion more than ORX. Further studies are necessary to discuss a relationship or interaction between the role of male hormone and fructose ingestion in lipid metabolism.

\section{Acknowledgements}

The present study was supported by a research fund for students and teachers from Ehime University and a research grant from the Japanese Ministry of Education, Science and Culture (16580101). There are no conflicts of interest. The contribution of each author is as follows: S. M. carried out the experimental plan, summarised the experimental results and discussed with the other researchers about the experimental results; T. K. advised all aspects of the experiment; K. E. designed the experiment and was involved in manuscript preparation.

\section{References}

1. Gevers Leuven JA (1994) Sex steroids and lipoprotein metabolism. Pharmacol Ther 64, 99-126.

2. Girard A, Madani S, Boukortt F, et al. (2006) Fructoseenriched diet modifies antioxidant status and lipid metabolism in spontaneously hypertensive rats. Nutrition 22, 758-766.

3. Bantle JP, Raatz SK, Thomas W, et al. (2000) Effects of dietary fructose on plasma lipids in healthy subjects. Am J Clin Nutr 72, 1128-1134.

4. Guthrie JF \& Morton JF (2000) Food sources of added sweeteners in the diets of Americans. J Am Diet Assoc 100, 43-51.

5. Bantle JP (2006) Is fructose the optimal low glycemic index sweetener? Nestle Nutr Workshop Ser Clin Perform Programme 11, 83-95.

6. Tappy L \& Lê KA (2010) Metabolic effects of fructose and the worldwide increase in obesity. Physiol Rev 90, 23-46.

7. Reeves PG, Nielsen FH \& Fahey GC (1993) AIN-93 purified diets for laboratory rodents: Final Report of the American Institute of the Nutrition $A d$ boc Writing Committee on the Reformulation of AIN-76A Rodent Diet. J Nutr 123, 1939-1951.

8. Folch J, Lees M \& Sloane Stanley GH (1957) A simple method for the isolation and purification of total lipides from animal tissues. J Biol Chem 226, 497-509.

9. Ebihara K, Shiraishi R \& Okuma K (1998) Hydroxypropylmodified potato starch increases fecal bile acid excretion in rats. J Nutr 128, 848-854. 
10. Sheltaway MJ \& Losowsky MS (1975) Determination of fecal bile acids by an enzymic method. Clin Chem Acta 64 , 127-132.

11. Ogishima T \& Okuda K (1986) An improved method for assay of cholesterol $7 \alpha$-hydroxylase activity. Anal Biochem 158, 228-232.

12. Chomczynski P \& Sacchi N (1987) Single-step method of RNA isolation by acid guanidinium thiocyanate-phenolchloroform extraction. Anal Biochem 162, 156-159.

13. Borst SE \& Conover CF (2006) Orchiectomized Fischer 344 male rat models body composition in hypogonadal state. Life Sci 79, 411-415.

14. Erben RG, Eberle J, Stahr K, et al. (2000) Androgen deficiency induces high turnover osteopenia in aged male rats: a sequential histomorphometric study. J Bone Miner Res 15, 1085-1098.

15. Asarian L \& Geary N (2006) Modulation of appetite by gonadal steroid hormones. Phil Trans R Soc B 361, 1251-1263.

16. Woods SC, Gotoh K \& Clegg DJ (2003) Gender differences in the control of energy homeostasis. Exp Biol Med 228, 1175-1180.

17. Chai JK, Blaha V, Meguid MM, et al. (1999) Use of orchiectomy and testosterone replacement to explore meal numberto-meal size relationship in male rats. Am J Physiol 276, R1366-R1373.

18. Hengge UR, Stocks K, Wiehler H, et al. (2003) Double-blind, randomized, placebo-controlled phase III trial of oxymetholone for the treatment of HIV wasting. AIDS 17, 699-710.

19. Morley JE (2001) Decreased food intake with aging. J Gerontology 56, A81-A88.

20. De Pergola G (2000) The adipose tissue metabolism: role of testosterone and dehydroepiandrosterone. Int J Obes Relat Metab Disord 24, S59-S63.

21. Song YM, Sheu WH, Lee WJ, et al. (1999) Plasma leptin concentrations are related to body fat mass and gender but not to thyroid dysfunction. Kaohsiung J Med Sci 15, 119-126.

22. Suga A, Hirano T, Kageyama H, et al. (2000) Effects of fructose and glucose on plasma leptin, insulin, and insulin resistance in lean and VMH-lesioned obese rats. Am J Physiol Endocrinol Metab 278, E677-E683.

23. Pinilla L, Seoane LM, Gonzalez L, et al. (1999) Regulation of serum leptin levels by gonadal function in rats. Eur $J$ Endocrinol 140, 468-473.

24. Castrogiovanni D, Perelló M, Gaillard RC, et al. (2003) Modulatory role of testosterone in plasma leptin turnover in rats. Endocrine 22, 203-210.

25. Margetic S, Gazzola C, Pegg GG, et al. (2002) Leptin: a review of its peripheral actions and interactions. Int J Obes Relat Metab Disord 26, 1407-1433.

26. Hirsch E \& Walsh M (1982) Effect of limited access to sucrose on overeating and patterns of feeding. Physiol Behav 29, 129-134.

27. Kanarek RB \& Marks-Kaufman R (1979) Developmental aspects of sucrose-induced obesity in rats. Physiol Behav 23, 881-885.

28. Bouchard C, Després JP \& Mauriège P (1993) Genetic and nongenetic determinants of regional fat distribution. Endocr Rev 14, 72-93.

29. Basciano H, Federico L \& Adeli K (2005) Fructose, insulin resistance, and metabolic dyslipidemia. Nutr Metab (Lond) 21, $2-5$.
30. Lane MD \& Cha SH (2009) Effect of glucose and fructose on food intake via malonyl-CoA signaling in the brain. Biochem Biophys Res Commun 382, 1-5.

31. Teff KL, Elliott SS, Tschöp M, et al. (2004) Dietary fructose reduces circulating insulin and leptin, attenuates postprandial suppression of ghrelin, and increases triglycerides in women. J Clin Endocrinol Metab 89, 2963-2972.

32. Haug A \& Hostmark AT (1999) Hypercholesterolemia, hypotriacylglycerolaemia and increased lipoprotein lipase activity following orchidectomy in rats. Acta Endocrinol (Copenh) 113, 133-139.

33. Ginsberg HN (1995) Synthesis and secretion of apolipoprotein B from cultured liver cells. Curr Opin Lipidol 6, 275-280.

34. Wetterau JR, Aggerbeck LP, Bouma ME, et al. (1992) Absence of microsomal triglyceride transfer protein in individuals with abetalipoproteinemia. Science 258, 999-1001.

35. Simon D, Charles MA, Nahoul K, et al. (1997) Association between plasma total testosterone and cardiovascular risk factors in healthy adult men: the telecom study. J Clin Endocrinol Metab 82, 682-685.

36. Traish AM, Abdou R \& Kypreos KE (2009) Androgen deficiency and atherosclerosis: the lipid link. Vascul Pharmacol 51, 303-313.

37. Rudling MH, Eggertsen OG \& Angelin B (1996) Regulation of rat hepatic low density lipoprotein receptors. In vivo stimulation by growth hormone is not mediated by insulin-like growth factor I. J Clin Invest 97, 292-299.

38. Améen C \& Oscarsson J (2003) Sex difference in hepatic microsomal triglyceride transfer protein expression is determined by the growth hormone secretory pattern in the rat. Endocrinology 144, 3914-3921.

39. Sinha YN, Selby FW, Lewis UJ, et al. (1972) Studies of GH secretion in mice by a homologous radioimmunoassay for mouse GH. Endocrinology 91, 784-792.

40. Strauch G, Pandos P \& Bricaire H (1971) Fructose induced growth hormone release. J Clin Endocrinol Metab 32 582-584.

41. Améen C, Lindén D, Larsson B-M, et al. (2004) Effects of gender and $\mathrm{GH}$ secretory pattern on sterol regulatory element-binding protein-1c and its target genes in rat liver. Am J Physiol Endocrinol Metab 287, E1039-E1048.

42. Miyazaki M, Dobrzyn A, Man WC, et al. (2004) Stearoyl-CoA desaturase 1 gene expression is necessary for fructosemediated induction of lipogenic gene expression by sterol regulatory element-binding protein-1c-dependent and -independent mechanisms. J Biol Chem 279, 25164-25171.

43. Parini P, Angelin B \& Rudling M (1999) Cholesterol and lipoprotein metabolism in aging: reversal of hypercholesterolemia by growth hormone treatment in old rats. Arterioscl Thromb Vasc Biol 19, 832-839.

44. Hallfrisch J (1990) Metabolic effects of dietary fructose. FASEB J 4, 2652-2660.

45. Ostos MA, Recalde D, Baroukh N, et al. (2002) Fructose intake increases hyperlipidemia and modifies apolipoprotein expression in apolipoprotein AI-CIII-AIV transgenic mice. J Nutr 132, 918-923.

46. Fields M \& Lewis CG (1999) Dietary fructose but not starch is responsible for hyperlipidemia associated with copper deficiency in rats: effect of high-fat diet. J Am Coll Nutr 18, $83-87$. 